\title{
Effect of discretization method of a computational domain on particle separation in a cyclone separator
}

\author{
Marek Wasilewski ${ }^{1, *}$, and Grzegorz Ligus ${ }^{2}$ \\ ${ }^{1}$ Opole University of Technology, Faculty of Production Engineering and Logistics, 76 Proszkowska \\ St., 45-758 Opole, Poland, \\ ${ }^{2}$ Opole University of Technology, Faculty of Mechanical Engineering, 76 Proszkowska St., \\ 45-758 Opole, Poland
}

\begin{abstract}
This paper reports the results of a study into the effect of the selection of a method of discretization of the computational domain on the solid particles separation process in a cyclone separator. The selection of an optimum discretization method to be implemented in a computational domain forms a particularly important task during analysis using CFD. The process of discretization largely affects the precision of the results gained using the CFD models as well as independence of the results on the mesh density and duration of the computations. The comparative analysis was performed on the basis of the results of experimental and numerical research with regard to the separation efficiency and pressure drop. It was observed that the computational domains generated on the basis of hexagonal meshes provide the best conformity of the results in terms of the separation efficiency, which is the key parameter applied to assess the quality of a cyclone separator.
\end{abstract}

\section{Introduction}

Cyclone separators form an element of a variety of industrial installations. They are considered to belong to the group of mechanical separators. Their role is concerned with the separation of solid particles from the fluid that carries them in an industrial process. The operating principle of a cyclone separator makes use of a centrifugal force; hence, the design of such separators is simple, production and exploitation costs are low and the apparatus is almost maintenance-free. In addition, they find application in extreme working conditions (i.e., in a wide range of pressures and temperatures), which enables designers to adapt them to the requirements of a given installation.

The research dealing with the flow inside cyclone separators is conducted using the following approaches: experimental testing [e.g. 1-4], application of analytical models [e.g. 5-8] and the use of numerical flow simulations (Computational Fluid Dynamics-CFD) [e.g. 9-17]. The greatest reliability is offered by the research performed by the application of two of the above methods performed concurrently with the purpose with solving

\footnotetext{
* Corresponding author: m.wasilewski@po.opole.pl
} 
a specific problem. For many years, the most common approach involves the use of experimental research. This type of study offers the necessary conditions to obtain the results of the actual flow of two-phase mixtures with the best accuracy. Since the turn of the centuries, this type of study is supported and enhanced by the results of methods based on CFD. The origins of the CFD method date back to 1950s [18]. On the basis of the universal momentum, mass and energy conservation laws, we are able to develop process models in order to enable the simulation of a variety of single- and multi-phase flows. However, the key role in this context is played by the adequate defining of a strategy to be applied throughout the numerical study. The selection of adequate computational models, optimum discretization of the computational domain and the identification of relevant boundary conditions provide the foundation on which reliable results can be obtained later on serving for the purposes of qualitative and quantitative analysis.

\section{Methodology and scope of the study}

The objective of this paper is to conduct an study into the effect of the discretization of a computational domain on the analysis of the phenomenon of solid particle separation in cyclone separators. The study applied two methods: experiments involving testing of separation efficiency performed using an experimental set-up and a numerical modeling of the flow. The results found by use of the first method were subsequently applied to conduct further analyses. The verification was performed with regard to the separation efficiency of the solid particles and pressure drop.

\subsection{Experimental research}

The experiments were conducted using the experimental setup in Fig. 1. The set-up comprises three basic elements: model of a cyclone separator, an air exhaust fan including an inverter (allowing a wide range of fan speed control) and a feeding system with solid particle dispenser with a device to control the mass flow rate of solid particles. The setup also contains measurement devices (flowmeter, differential pressure transducer and anemometer). In addition, a granulometer was installed to provide the analysis of solid particle separation with the purpose of determining the efficiency of the specific fraction separation. The study applied a model of a cyclone separator with a helical roof inlet. The research was performed for a constant value of the mass flow rate of gas $\left(\mathrm{Qi}_{\mathrm{n}}=5.58 \mathrm{~m}^{3} / \mathrm{s}\right.$; $\left.\mathrm{v}_{\text {in }}=16 \mathrm{~m} / \mathrm{s}\right)$ and a mass flow rate of solid particles $\left(\mathrm{G}_{\mathrm{in}}=0.18 \mathrm{~kg} / \mathrm{s}\right)$.
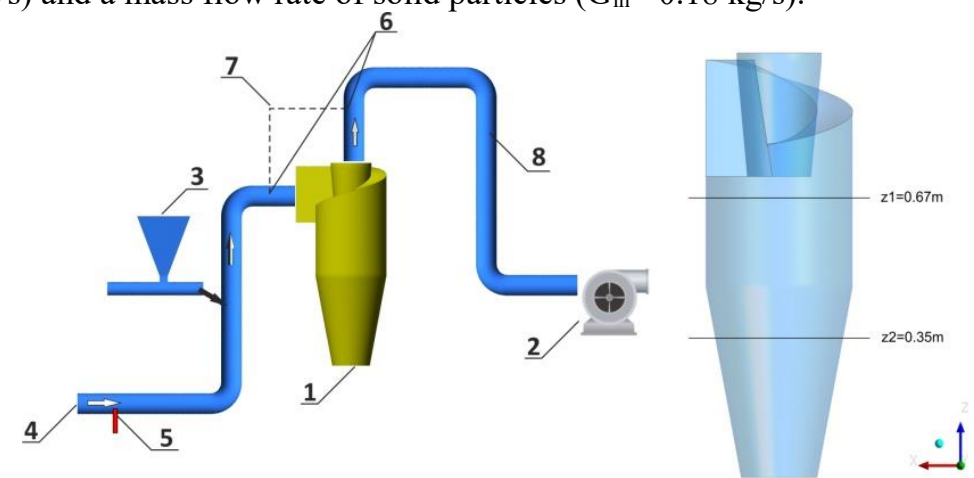

Fig. 1. Schematic of the experimental setup; 1. Cyclone separator; 2. Air exhaust fan; 3. Feeding system; 4. Inlet pipe; 5. Flow meter; 6. Pressure sensors; 7. Differential pressure transducer; 8. Outlet pipe. 


\subsection{CFD studies}

The majority of flows encountered in chemical and process engineering take the form of the turbulent flow. Such flows are characterized by irregularity, formation of vortices, diffusion and non-continuity. The existence of vortices affects an increase of the efficiency of mass and momentum exchange. However, all of this also leads to an increase of the friction factor and pressure drop. Hence, the use of the CFD methods involves the need to model the phenomena concerned with turbulent flows. The most common of the approaches is based on the use of Reynolds average Navier-Stokes method (RANS). The approach in this method applies the representation of the components of the velocity vectors and remaining scalar functions (in this case pressure) in terms of the mean value and its fluctuations. This equation can be written as $[10,19-21]$ :

$$
\begin{gathered}
\frac{\partial \mathrm{u}_{i}}{\partial x_{i}}=0 \\
\rho u_{j} \frac{\partial \mathrm{u}_{i}}{\partial x_{j}}=-\frac{\partial P}{\partial x_{i}}+\frac{\partial}{\partial x_{j}}+\left[\mu\left(\frac{\partial \mathrm{u}_{i}}{\partial x_{j}}+\frac{\partial \mathrm{u}_{j}}{\partial x_{i}}\right)\right]+\frac{\partial \tau_{i j}}{\partial x_{j}}
\end{gathered}
$$

A solution to this issue requires the statement of a closure hypothesis. Such a step provides an equal number of unknowns with the number of independent equations. Reynolds Stress Model (RSM) forms the most common closure model for the case of cyclone separators [e.g. 9, 10, 14, 16, 19]. This model can be given by the following equation $[14,20,21]$ :

$$
\frac{\partial}{\partial t}\left(\rho \bar{u}_{\imath}^{\prime} \bar{u}_{\jmath}^{\prime}\right)+\frac{\partial}{\partial x_{k}}\left(\rho u_{k} \overline{u_{\imath}^{\prime}} \overline{u_{\jmath}^{\prime}}\right)=D_{i j}+P_{i j}+\Pi_{i j}+\varepsilon_{i j}+s
$$

\subsubsection{Discretization of computational domain}

The discretization of computational domain involves the transformation of the initial transport equations into algebraic equations approximating them by defining the relations of the dependent variables in the selected regions in time and space. One of the most common approach to discretization applies the use of the mesh method. As a result of discretization, the differential equations (in which each one is linearized) is replaced by a system of algebraic equations, whose consequence takes the form a linear algebraic equations. This process occurs by discretization of a computational mesh. Each of the elements of this mesh corresponds to a control volume throughout the calculations. Depending on the discretization method, the mesh is composed of nodes, elements or volumes. The level of the precision gained from the solution of numerical problems is largely relative to the type and density of such a mesh.

Depending on the applied geometrical form, meshes can be classified into triangular and quadrilateral (for two-dimensional models). In turn, for three-dimensional models, the most common meshes are tetrahedral (with a base in the form of a triangle), pyramid-shaped prisms with a triangular base, and hexagonal prism with a base in the form of a triangle. The use of hexagonal cells results in the limitation of the number of the mesh elements in the computational domain and provides a better accuracy of calculations. However, the generation of this type of meshes requires a considerable input in terms of amount of required effort. In turn, tetrahedral meshes are applied with regard to models with considerable curvatures and in cases when numerous details need to be accounted for. 
In such as case a number of post-processors have the capability of generating this type of mesh.

The classification in terms of the arrangement of the cells in a mesh, we can distinguish structured, unstructured and hybrid meshes. In the first type, cells are characterized by regular connectivity. The generation of this mesh type requires more effort; hence, they are recommended for the case of simple geometries. The unstructured type is applied most commonly for the case of complex geometries. In this case, any shape of each mesh cell can be used. In many cases, the third type of cell arrangement can provide useful. It combines the advantages of structured and unstructured meshes.

\subsubsection{Description of CFD research and discretization method}

A model was developed using the geometry of the cyclone separator used in the experimental study. A three-dimensional domain was applied for this purpose. In a further step, assumptions were adopted with regard to the investigated alternatives of discretization of the computational domain. Due to the scope of the researched problem, the analysis involved three alternatives of the mesh design-tetrahedral (alternative a), hexagonal (alternative b) and mixed (comprising tetrahedral cells in the upper region - with the complex geometry and hexagonal in the lower region-alternative c). In addition, due to the fact that the accuracy of the results and computational time is largely affected by the mesh density, three alternatives of mesh density were adopted (marked as 1, 2, 3, respectively). As a consequence, nine computational domains were developed and subsequently applied in the procedure. In order to provide an adequate quality of the adopted meshes, the mean value of skewness was taken to be equal to a maximum of 0.25 . Table 1 contains a specification of the analyzed alternatives, including the values of the mean skewness. Fig. 2 presents three examples computational meshes generated during the present study.

The analysis of the flows was performed by the application of the finite volume method. The selected approach of solving was based on the statement of pressure-based pressures equations. The solution of the differential equations utilized the SIMPLE algorithm, with the purpose of adequate statement of the coupling of the pressure and velocity fields for maintaining continuity of momentum curves. The closure model applied RSM model. The second-order upwind interpolation method was used to determine the representative samples of the constituent values on the surface of the control volumes. The condition of convergence for the continuity equation was set at $10^{-6}$, whereas for the remaining equations, it was $10^{-3}$. The flow conditions corresponded to the conditions applied during the experimental research $\left(\mathrm{V}_{\text {in }}, \mathrm{Q}_{\text {in }}, \mathrm{G}_{\text {in }}\right.$ ). The boundary condition for the inlet was applied to be 'velocity-inlet', while for the outlet, it was 'outflow'. The reflect boundary conditions were used for the walls. The representation of the presence of the continuous phase applied the Euler-Lagrange method. In this case, fluid is considered as a continuous phase, whereas the dispersed phase (solid phase) is identified through the particle tracking. 
Table 1. Characteristic of the analyzed computational domains.

\begin{tabular}{|c|c|c|c|}
\hline $\begin{array}{c}\text { Variants of } \\
\text { meshes }\end{array}$ & $\begin{array}{c}\text { Total number of } \\
\text { elements }\end{array}$ & Total number of nodes & $\begin{array}{c}\text { Average } \\
\text { skewness }\end{array}$ \\
\hline Mesh a1 & 593629 & 105975 & 0.173 \\
\hline Mesh a2 & 756378 & 134405 & 0.168 \\
\hline Mesh a3 & 983536 & 173760 & 0.165 \\
\hline Mesh b1 & 191702 & 183908 & 0.156 \\
\hline Mesh b2 & 312635 & 301667 & 0.143 \\
\hline Mesh b3 & 411552 & 395231 & 0.139 \\
\hline Mesh c1 & 420571 & 160524 & 0.192 \\
\hline Mesh c2 & 473101 & 179923 & 0.188 \\
\hline Mesh c3 & 716725 & 269168 & 0.178 \\
\hline
\end{tabular}

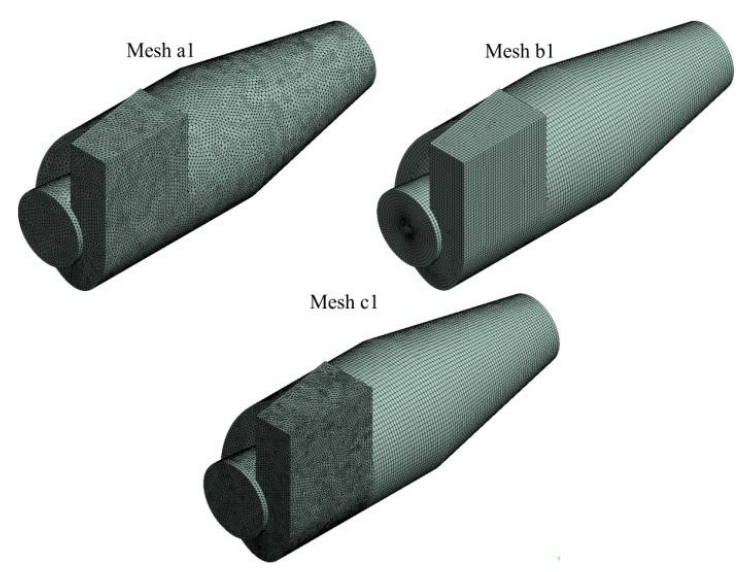

Fig. 2. Examples of computational domains.

\section{Results and discussion}

The efficiency of the separation was the principal parameter analyzed in this paper. This parameter plays a crucial role during the selection of a geometry of a cyclone separator at the phase of the design of an installation. The values gained from the experiments served as a source of reference for the results obtained throughout numerical calculations, as the efficiency of the former case was equal to $92.3 \%$. Table 2 contains a summary of the results. Beside the total separation efficiency, this table also contains data regarding the fractional efficiency for the particular solid particle diameters. By analyzing these values, we can observe that the best conformity was gained for the computational domains generated by the application of hexagonal meshes. In addition, along with the increase of their density, the efficiency of separation increases slightly. The value of the error for this type of meshes is the range from $2.4 \%$ to $2.8 \%$. For the case of domains applying tetrahedral meshes, this error is in the range 3\% to 5.4\%. An increase of the density of meshes has a negative effect on the results gained with regard to the separation efficiency. An identical tendency was observed with regard to mixed meshes (i.e. error is in the range from $3.5 \%$ to $5 \%$ ). On the basis of the analysis of separation efficiency of the particular fractions, we can observe that the key role is played by the efficiency of separation for the particles with the diameters of $15 \mu \mathrm{m}$ and $30 \mu \mathrm{m}$. For the case of the particle with the $15 \mu \mathrm{m}$ diameter, the best efficiency was recorded as a result of using the analyzed a1 and b3 alternatives. Concurrently, for the particles with the $30 \mu \mathrm{m}$ diameter, 
we can clearly note an increase of the efficiency for all domains as a result of applying hexagonal meshes (the best conformity was gained for the b3 alternative) in comparison to other domains. The lowest values for both diameters was gained for the a3 and c3 alternatives. For the particle diameters above $90 \mu \mathrm{m}$, the efficiency of solid particle separation was $100 \%$ in all cases.

By analyzing the second parameter applied to assess the efficiency of cyclone separators. namely pressure drop. we can note that most accurate results were gained using the computational domains with a mixed structure (i.e. error was in the range from $3.3 \%$ to $4.9 \%)$. The maximum conformity was gained for the c3 alternative $(96.7 \%)$. It is noteworthy that for the hexagonal domains the compatibility of the results was also high (i.e. error was in the range from $6.4 \%$ to $6.7 \%$ ). The worst accuracy was recorded in the cases when tetrahedral domains were applied (i.e. error was in the range from $11.9 \%$ to $13.2 \%)$.

Table 2. Efficiency of solid particle separation and values of pressure drop for the analyzed alternatives.

\begin{tabular}{|c|c|c|c|c|c|c|c|c|c|c|}
\hline $\begin{array}{c}\text { Particle } \\
\text { diameter }[\boldsymbol{\mu m}]\end{array}$ & $\begin{array}{c}\text { Exp. } \\
\text { Result }\end{array}$ & $\mathbf{9 1}$ & $\mathbf{a 2}$ & $\mathbf{a 3}$ & $\mathbf{b 1}$ & $\mathbf{b 2}$ & $\mathbf{b 3}$ & $\mathbf{c 1}$ & $\mathbf{c 2}$ & $\mathbf{c 3}$ \\
\hline $\mathbf{1 5}$ & $76.0 \%$ & $71.5 \%$ & $68.3 \%$ & $64.9 \%$ & $67.8 \%$ & $68.8 \%$ & $69.0 \%$ & $68.0 \%$ & $67.1 \%$ & $65.7 \%$ \\
\hline $\mathbf{3 0}$ & $94.0 \%$ & $89.9 \%$ & $89.1 \%$ & $88.1 \%$ & $92.2 \%$ & $92.3 \%$ & $92.5 \%$ & $90.8 \%$ & $89.6 \%$ & $88.8 \%$ \\
\hline $\mathbf{4 5}$ & $96.0 \%$ & $92.5 \%$ & $92.1 \%$ & $91.4 \%$ & $95.8 \%$ & $95.2 \%$ & $95.6 \%$ & $93.7 \%$ & $92.5 \%$ & $91.9 \%$ \\
\hline $\mathbf{6 0}$ & $98.0 \%$ & $94.1 \%$ & $93.8 \%$ & $93.2 \%$ & $96.6 \%$ & $96.1 \%$ & $96.5 \%$ & $95.0 \%$ & $94.1 \%$ & $93.4 \%$ \\
\hline $\mathbf{9 0}$ & $100.0 \%$ & $100.0 \%$ & $100.0 \%$ & $100.0 \%$ & $100.0 \%$ & $100.0 \%$ & $100.0 \%$ & $100.0 \%$ & $100.0 \%$ & $100.0 \%$ \\
\hline $\mathbf{1 1 0}$ & $100.0 \%$ & $100.0 \%$ & $100.0 \%$ & $100.0 \%$ & $100.0 \%$ & $100.0 \%$ & $100.0 \%$ & $100.0 \%$ & $100.0 \%$ & $100.0 \%$ \\
\hline $\mathbf{1 4 0}$ & $100.0 \%$ & $100.0 \%$ & $100.0 \%$ & $100.0 \%$ & $100.0 \%$ & $100.0 \%$ & $100.0 \%$ & $100.0 \%$ & $100.0 \%$ & $100.0 \%$ \\
\hline $\mathbf{2 0 0}$ & $100.0 \%$ & $100.0 \%$ & $100.0 \%$ & $100.0 \%$ & $100.0 \%$ & $100.0 \%$ & $100.0 \%$ & $100.0 \%$ & $100.0 \%$ & $100.0 \%$ \\
\hline $\begin{array}{c}\text { Overall } \\
\text { separation } \\
\text { efficiency [\%] }\end{array}$ & $92.3 \%$ & $89.5 \%$ & $88.4 \%$ & $87.2 \%$ & $89.7 \%$ & $89.8 \%$ & $90.0 \%$ & $89.0 \%$ & $88.3 \%$ & $87.7 \%$ \\
\hline Press. drop [Pa] & 1419 & 1613 & 1610 & 1634 & 1521 & 1515 & 1516 & 1350 & 1361 & 1372 \\
\hline
\end{tabular}

The analysis of the local velocities inside the cyclone separator was undertaken so as to offer an interpretation to the observed tendencies. This analysis applied two crosssections $\mathrm{z} 1=0.67 \mathrm{~m}$ and $\mathrm{z} 2=0.35 \mathrm{~m}$. Fig. 3 contains the results of the local velocities in both cross-sections. For the case of local velocities along the $\mathrm{z} 1$ cross-sections (Fig. 3a). maximum values were recorded for all alternatives in the axis of the cyclone separator. This resulted from an increase of the turbulence of the flow in the entire region (i.e. the bottom region of the vortex finder). In this region. the values of the velocities for the domains with the tetrahedral structure were considerably different from the remaining alternatives. The considerably greater velocity led to an increase of the pressures drop and entrainment of greater amounts of unseparated solid particles entrained into the vortex finder. For the remaining domains. very similar values were gained. For the case of the z2 position (Fig. 3b) the smallest velocities were gained along the axis of the cyclone separator. This is due to the formation of the internal vortex entraining gas in the lower section of the separator. which is characterized by smaller velocities compared to the external vortex.

Fig. $3 \mathrm{c}$ and $3 \mathrm{~d}$ present the tangential velocities - which form the dominant element of the gas flow in the cyclone and affect the centrifugal force (as this force increases along with the increase of the velocity). For this reason. tangential velocity has a considerable influence on the pressure drop and separation capabilities of the cyclone separator. Since pressure and velocity are mutually interdependent. we can foresee that the cyclone designs with greater static pressure produce stronger tangential velocity fields. The tangential velocity fields can be separated into two regions. i.e. an outer free downward vortex and an upward vortex called the 'Rankine vortex'. For the case of tangential velocities, the greatest 
values are gained for the domains with tetrahedral meshes and these values differ from the ones for remaining two domains. We can also emphasize here that the increase of the mesh density slightly affects the change in the velocities. For the case of hexagonal meshes, only the case with the b1 alternative provided distinct results from the two remaining mesh densities - b2 and b3. For the domains with a mixed structure. the change in the velocity also has a small effect on the values that were recorded. This information can be useful during further analysis into the susceptibility of the mesh on the results in cyclone separators. In this context. research needs to be conducted so as to determine the density for which a similarity of the results gained from computational models and experimental research is gained. The results can be applied to offer a limitation to the excessive increase of the density of computational meshes applied in research. The results demonstrate that not in all cases an increase of the mesh density can offer better results.

This comparative analysis can be supplemented by Fig. 4, in which the contours of the velocity (fig. 4 a) and velocity vectors (fig. 4 b) are presented in the longitudinal crosssections of the cyclone separator for the alternatives for which the maximum (a3) and minimum values of the velocity (c3) were gained. We can note considerable differences between the values found there, which are also clear by analyzing the values of the registered pressure drop and efficiency of the separation. In both cases, there is a local increase of the flow turbulence in the vicinity of the bottom region of the vortex finder.
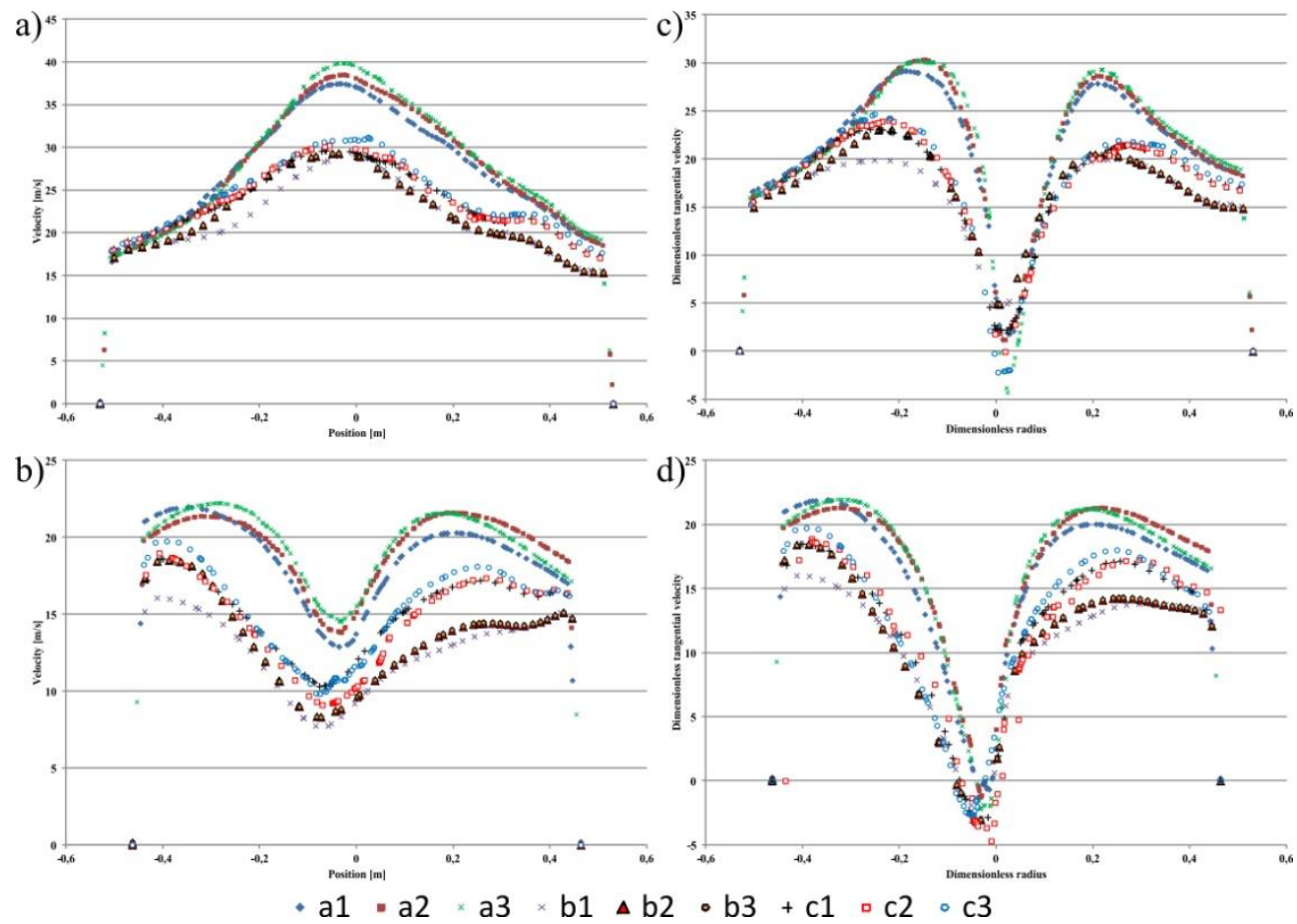

Fig. 3. Radial profiles of velocity in the locations z1 (a) and z2 (b) and profiles of tangential velocity in the locations z1 (c) and z2 (d). 

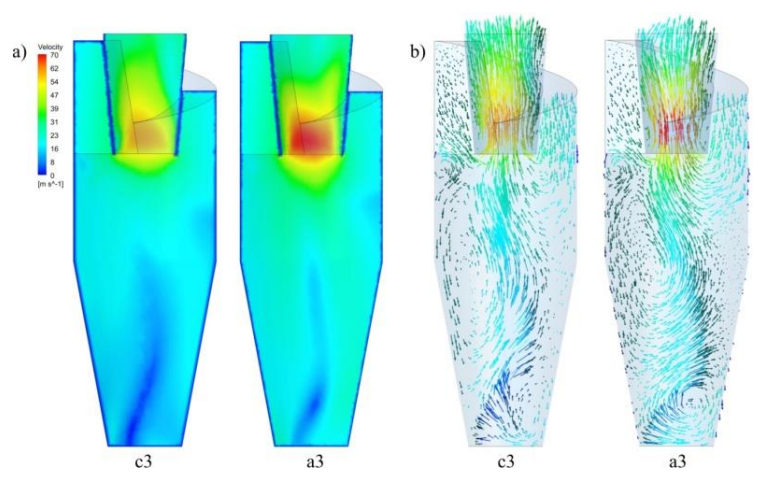

Fig. 4. Contours of the velocities (a) and velocity vectors (b) along the transverse cross-section of the separator for $\mathrm{c} 3$ and $\mathrm{a} 3$ alternatives.

\section{Conclusions}

The analysis conducted here leads to the statement that for the case of cyclone separators. it is justified to apply computational meshes using hexagonal meshes. The values of the separation efficiency (i.e. the key parameter which is used to describe the quality of a cyclone separator) gained in this study for such configurations of computational domains demonstrated the best conformity with the results of experiments. Besides. with regard to the other parameter - that is pressure drop - the results were characterized by satisfactory conformity (and slightly smaller than for the case of domains with mixed structure). The use of hexagonal meshes involves a greater input of labor associated with the configuration of the computational domains and longer duration of the computations. Hence. it is important to initially take into consideration the characteristics of the studies that are to be undertaken. For the case of research industrial (when a shorter computational time is the key aspect). the selection of a domain with the smaller computational requirements is justified. However. the conditions of scientific studies and access to highend equipment with high performance computers justifies the longer duration of the research aimed at gaining precise results.

This research was carried out with the support of the Interdisciplinary Centre for Mathematical and Computational Modelling (ICM) University of Warsaw under grant no G71-5.

\section{References}

1. Y. Zhu. K.W. Lee, J. Aerosol Sci. 30 (1999)

2. A.C. Hoffmann. M. De Groot. W. Peng. H.W.A. Dries. J. Kater, AIChE J. 47 (2001)

3. W. Peng. A.C. Hoffmann. H.W.A. Dries. M.A. Regelink. L.E. Stein, Chem. Eng. Sci. 60 (2005)

4. W. Li. J. Chen, Front. Mech. Eng. China, 2 (2007)

5. R.M. Alexander, The Australasian Institute of Mining and Metallurgy, 152 (1949)

6. C.J. Stairmand, Ind. Eng. Chem. 29 (1951)

7. E. Muschelknautz, Chem. Ing. Tech. 44 (1972)

8. A. Avci. I. Karagoz, Heat Mass Transfer, 28 (2001) 
9. H. Safikhani. M. Akhavan-Behabadi. M. Shams. M.H. Rahimyan, Adv. Powder Technol. 21 (2010)

10. M. Azadi. M. Azadi. A. Mohebbi, J. Hazard Mater. 182 (2010)

11. L.S. Brar. R.P. Sharma. K. Elsayed, Powder Technol. 286 (2015)

12. C.W. Haig. A. Hursthouse. D. Sykes. S. Mcilwain, Appl. Math. Model. 40 (2016)

13. J.J.H. Houben. S. Pirker, J. Appl. Fluid. Mech. 9 (2016)

14. M. Wasilewski, Sep. Purif. Techol. 168 (2016)

15. K.W.Chu. J.Chen. B.Wang. A.B.Yu. A.Vince, Powder Technol. 320 (2017)

16. M. Wasilewski. L.S. Brar, Powder Technol. 313 (2017)

17. H. Mikulcic. M. Vujanovic. M. S. Ashhab. N. Duic, Energy, 75 (2014)

18. T.J. Chung, Computational Fluid Dynamics (Cambridge University Press, Cambridge, 2002)

19. J.P. Wu. Y.H. Zhang. H.L. Wang, Sep. Purif. Technol. 132 (2014)

20. Fluent User's Guide

21. B. Wang, D.L. Xu, K.W. Chu, A.B. Yu, App. Math. Model. 30 (2006) 